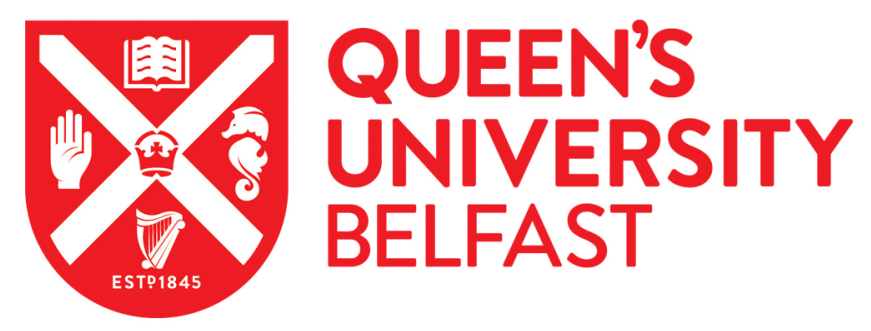

\title{
Supervised Non-Intrusive Load Monitoring algorithm for Electric Vehicle Identification
}

Moreno Jaramillo, A., Laverty, D., Hastings, J., Martinez-del-Rincon, J., \& Morrow, D. J. (2020). Supervised NonIntrusive Load Monitoring algorithm for Electric Vehicle Identification. In Proceedings of the 2020 IEEE International Instrumentation and Measurement Technology Conference (Proceedings of the IEEE International Instrumentation and Measurement Technology Conference). Institute of Electrical and Electronics Engineers Inc.. https://doi.org/10.1109//2MTC43012.2020.9128529

Published in:

Proceedings of the 2020 IEEE International Instrumentation and Measurement Technology Conference

\section{Document Version:}

Peer reviewed version

Queen's University Belfast - Research Portal:

Link to publication record in Queen's University Belfast Research Portal

Publisher rights

Copyright 2020 IEEE. This work is made available online in accordance with the publisher's policies. Please refer to any applicable terms of use of the publisher.

\section{General rights}

Copyright for the publications made accessible via the Queen's University Belfast Research Portal is retained by the author(s) and / or other copyright owners and it is a condition of accessing these publications that users recognise and abide by the legal requirements associated with these rights.

Take down policy

The Research Portal is Queen's institutional repository that provides access to Queen's research output. Every effort has been made to ensure that content in the Research Portal does not infringe any person's rights, or applicable UK laws. If you discover content in the Research Portal that you believe breaches copyright or violates any law, please contact openaccess@qub.ac.uk. 


\section{Supervised Non-Intrusive Load Monitoring Algorithm for Electric Vehicle Identification}

\author{
Andrés F. Moreno Jaramillo \\ School of Electronics, Electrical \\ Engineering and Computer \\ Science (EEECS) \\ Queen's University Belfast \\ Belfast, United Kingdom \\ amorenojaramillo01@qub.ac.uk
}

\author{
David M. Laverty \\ School of Electronics, Electrica \\ Engineering and Computer \\ Science (EEECS) \\ Queen's University Belfast \\ Belfast, United Kingdom \\ david.laverty@qub.ac.uk
}

\author{
Jesús Martínez del Rincón \\ School of Electronics, Electrical \\ Engineering and Computer \\ Science (EEECS) \\ Queen's University Belfast \\ Belfast, United Kingdom \\ j.martinez-del-rincon@qub.ac.uk
}

\author{
John Hastings \\ School of Electronics, Electrica \\ Engineering and Computer \\ Science (EEECS) \\ Queen's University Belfast \\ Belfast, United Kingdom \\ J.hastings@qub.ac.uk
}

\author{
D. John Morrow \\ School of Electronics, Electrica \\ Engineering and Computer \\ Science (EEECS) \\ Queen's University Belfast \\ Belfast, United Kingdom \\ Dj.Morrow@qub.ac.uk
}

\begin{abstract}
Transport sector electrification represents an increase in the number of electric vehicles (EV), producing significant variations in the distribution network dynamics. As a result, bidirectional power flow, overload and load unbalances are caused at the low voltage level due to unexpected increased load peaks. Non-intrusive load monitoring (NILM) methods have been developed as a strategy for energy management systems, applied to the customer side producing energy savings. This research presents a NILM methodology based on a low complexity conventional supervised machine learning pipeline. Our approach uses Principal Component Analysis (PCA) and Random Forest (RF) to detect the presence of a charging electric vehicle on the electricity network. By processing low sampling rate active power data, this approach provides a simple but feasible method that can be applied to smart meters. This provides useful data analysis for distribution network operators (DNO) to effectively deal with variability caused by these low carbon loads in the distribution grid. Achieving an overall efficacy of $92.68 \%$, the proposed method can be compared with other state of the art methods developed under higher complexity techniques.
\end{abstract}

Keywords-Discrete Wavelet Transformation, Electric Vehicle, Load Signature, Non-intrusive load monitoring, Random Forest.

\section{INTRODUCTION}

Throughout the world, governments have agreed policies and set targets to reduce carbon dioxide $\left(\mathrm{CO}_{2}\right)$ emissions caused by burning fossil fuels. Strategies to date have often focused on decarbonisation of the electricity sector, but the transportation sector accounts for $25 \%$ of global energy consumption [1], generating around a quarter of the global $\mathrm{CO} 2$ emissions [2]. Consequently, electric vehicles (EVs) are proposed as a sustainable alternative to conventional internal combustion engine vehicles.

Electrical distribution networks are faced with a number of challenges arising from the charging of EVs. Depending on factors such as geographical distribution, electric vehicle charging patterns and charging times among others, technical issues such as electrical system unbalances, coordination of protection problems, voltage instability, increased peak load, increased harmonic distortion and so on are caused by large scale implementation of EVs in the distribution grid [3].

In order to adapt to the challenges of EVs, DNOs must identify the presence of EVs on their network. Therefore, nonintrusive load monitoring (NILM) is one such technique contributing to energy management systems. Analysing aggregated measurements and decomposing electrical signals, individual load profiles can be identified. NILM methods can be categorized into two main groups: supervised and unsupervised algorithms. Supervised approaches use labelled data to generate a model, allowing the system to classify the output as known classes. Linear regression, Naïve Bayes, support vector machine (SVM), decision tree (DT), random forest $(\mathrm{RF})$ and $\mathrm{k}$-nearest neighbour $(\mathrm{kNN})$ are one of the most commonly used NILM supervised algorithms [4]. In contrast, non-supervised methods do not require labelling during training, classifying the input into clusters. Methods such as Kmeans, Hidden Markov Model (HMM) and its variations belong to this group [5].

In this paper, a low complexity NILM methodology is proposed using low sampling rate data, available from the public "Pecan Street - Dataport" dataset [6]. It is based on Principal Component Analysis (PCA) and Random Forest (RF) algorithms to identify electric vehicle load profiles from aggregated measurements in the residential sector. The main advantage consists on the implementation of conventional machine learning techniques to classify data recorded every minute, a typical sampling rate frequency of smart meters in the end customer side [5]. Thus, communication and processing requirements are minimized. Additionally, useful information for distribution network operators is provided to improve controllability and flexibility at the very end of the electrical grid where new low carbon loads have been integrated.

\section{WORK TO DATE}

Although most of the NILM algorithms have been developed to identify conventional loads based on analysis of electrical variables recorded during steady-state and transient conditions, there are some developments focused on the identification of non-conventional loads such as EVs. For instance, using active power measurements from the "Pecan Street - Dataport" dataset, A. Munshi and Y. Mohammed presented in [7] an unsupervised NILM methodology using ICA to obtain electric vehicles load signature, divided into three different sections: initial, steady and final charging stages. Using precision, recall and F1-score to evaluate the method, an efficacy of $86.05 \%$ was achieved. Additionally, a supervised event NILM method was developed by A. Rheman, T. Lie and S. Tio in [8]. Air conditioning and EV connection and disconnections were classified from segregated measurements using low sampling rate data. Mean sliding 
window (MSW) was implemented to extract transient features such as peak to peak power, transient time as well as statistical characteristics in the initial stage of the algorithm. These variables were used as inputs of a $\mathrm{kNN}$ classifier.

Another common approach is based on load modelling. In [9], an EV load forecasting method using deep learning algorithms based on experimental data acquired from a plug-in electric vehicles station in Shenzhen is presented. Performance of the system was evaluated with root mean squared error (RMSE), mean absolute percent error (MAPE) and mean absolute error (MAE) metrics, obtaining the performance from a long-short-term memory (LSTM) deep learning technique. However, bearing in mind the aim of this research is focused on the identification rather than the prediction, this approach was not considered.

\section{PROPOSED METHODOLOGY}

The aim of this research study is to develop a NILM methodology to identify EV load profiles from aggregated measurements in the residential sector from active power data acquired at low sampling rates. The structure of the proposed method can be separated in three main sections, namely data acquisition, data processing and EV classification as it is shown in Fig. 1.

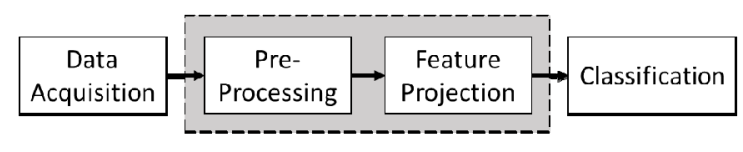

Fig. 1. Proposed NILM algorithm structure

\section{A. Data Acquisition}

"Pecan Street - Dataport", HELD1 [10], BLUED [11], UKDALE [12], REDD [13] are some of the most commonly used public datasets used by researchers to design NILM algorithms. Containing electrical variable measurements from over one thousand domestic properties distributed across Texas, Colorado, California and other places in the United States, Pecan Street Dataset is one of the highest public databases available for research purposes.

Active power measurements taken every minute recorded over a two-year period between 01/01/2016 and 31/12/2017 from 6 different domestic residences were considered. Additionally, 6 individual load signatures over the same period of time, coming from Chevrolet Volt, Nissan Leaf, Tesla S Model and a Mitsubishi i-MiEV were also used. For instance, in Fig. 2 it can be seen individual load profiles from furnace (purple line), electric vehicle (blue line) and the aggregated power consumption of the domestic property 1202 (red doted line), during the $5^{\text {th }}$ of February in 2016. From this graph, it can be said air-conditioning systems, dishwasher and the disposal where not used during the mentioned day, but other nonmonitored loads plugged into the house were contributing to have an average energy demand of $1.88 \mathrm{~kW} / \mathrm{h}$.

Grid signal representing total active power demand of the electrical appliances in the house as well as the vehicle charging profile have been selected as inputs to train and test the NILM algorithm.

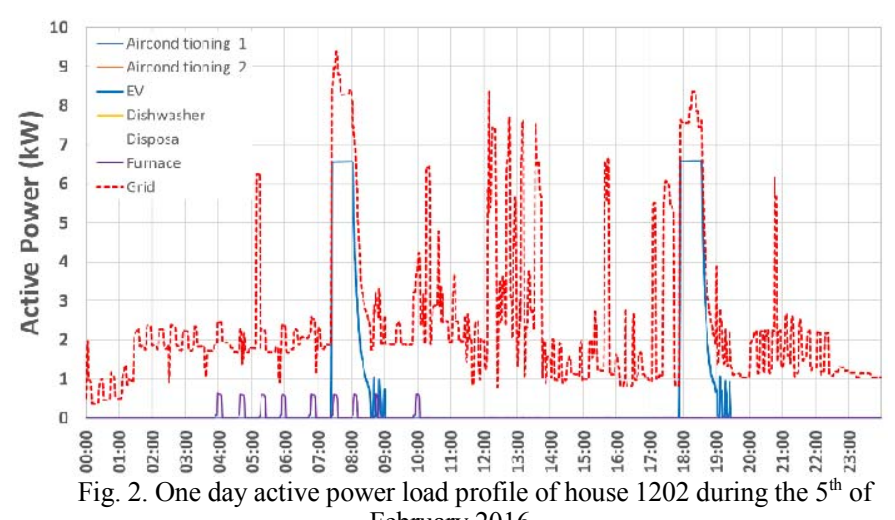

February 2016

\section{B. Pre-processing}

Pre-processing stage refers to the initial adequations required for the data to be used as input of the machine learning algorithms. In this case, sliding windows and data selection process are applied to the initial data acquired from the "Pecan Street - Dataport" dataset.

In order to analyse the data in shorter periods of time, sliding windows were used for to make the system adaptable under changes in the load. Setting the window width to 10 minutes enables the system to recognize initial charging time, constant charging period and final charge of each EV among the other household loads. Sliding windows were formed as shown in Fig. 3, to continuously monitor the presence of EV in the signal without losing information between transitions from one sample to another one.

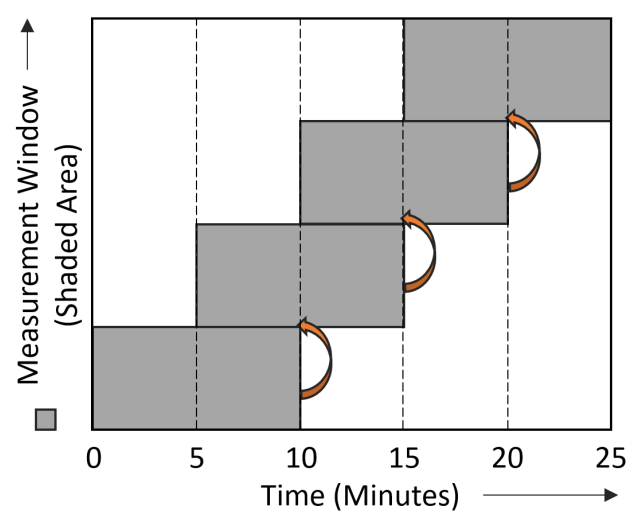

Fig. 3. Sliding window process

A total of 7.739 .784 windows were obtained in with only around $6 \%$ representing power consumption of both EV charging and conventional residential loads. The other $94 \%$ contained energy demand from only conventional household loads. Therefore, only $12 \%$ of the total dataset was used for training and testing purposes (all windows with electric vehicle loads plus an equivalent amount of windows with only conventional loads selected randomly among the rest), discarding $88 \%$ of the data containing only electrical consumption of conventional loads as it is shown in Fig. 4, obtaining a balanced dataset as a result. 


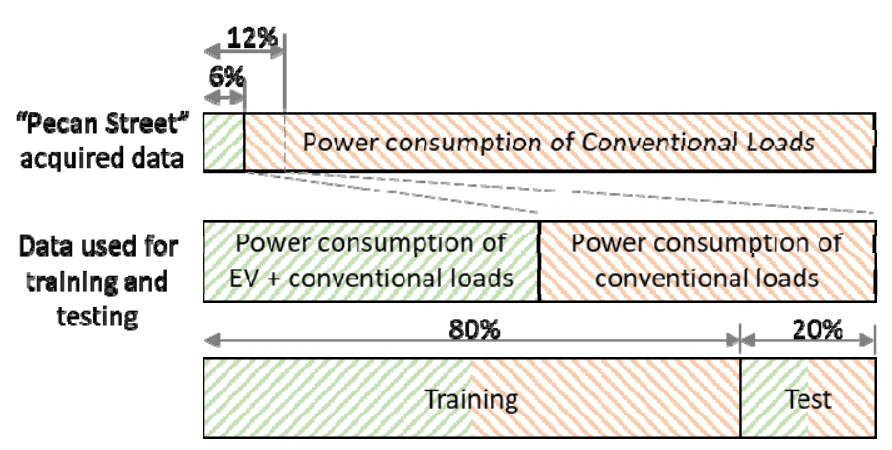

Fig. 4. Data selection for training and testing sets

\section{Feature Projection}

Extracted windows in Section B are subject to contain a certain degree of redundancy. Moreover, some of them may not be fully discriminative or affected by noise. To address these issues, feature dimensionality reduction or feature projection allows us to extract the relevant information from the feature vector. Considering the dimension of the input data, PCA is implemented as dimensionality reduction method to discard any repeated or unnecessary information. This contributes enhancing processing times as well as performance of the classification algorithm [14]. By applying PCA, the data, originally represented as time domain components (or dimensions) is projected to another dimensional space, whose dimensions are a set of orthogonal axis called principal components. By preserving only those principal components that contain most of the variance of the original data, it is possible to preserve the important information from the original data while reducing those components that are redundant or noisy [15].

To do so, the input data is pre-processed centring and scaling values of each window, setting the average and variance to 0 and 1 respectively. Subsequently, PCA loading scores and the variation each principal component are calculated. For this case, all principal components were used, thus 10 principal components where obtained. The expression shown in equation 1, represents the mathematical procedure to calculate the variation of each new component.

$$
\operatorname{var}=\frac{\left(\mathrm{x}_{\mathrm{i}}-\dot{\mathrm{X}}\right)^{2}}{\mathrm{n}}
$$

Where $\mathrm{x}_{\mathrm{i}}$ represents each measurement, $\dot{\mathrm{x}}$ represents the mean of the analysed window and $n$ is the total number of measurements per window, set as 10 in this case. Obtained variances from each principal component are displayed in Fig. 5.

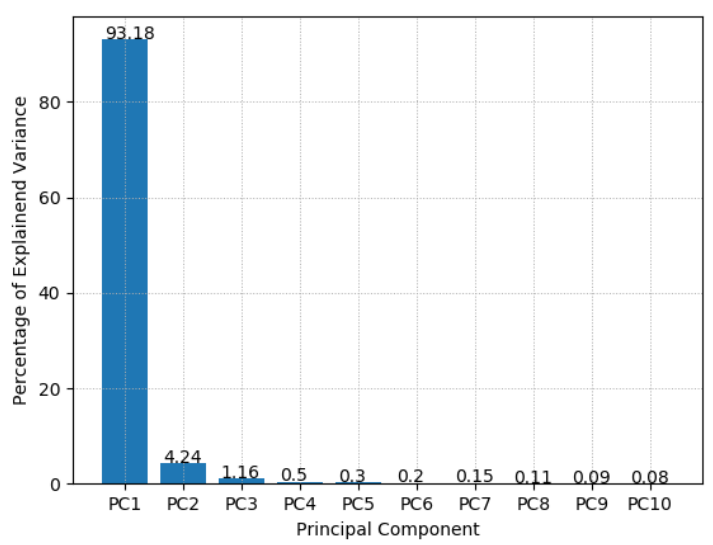

Fig. 5. Explained principal components variance

Based on the creation of eigenvectors where all points are projected, PCA reduces dimensionality of the input matrix containing the most valuable information to appropriately represent the input signal but eliminating redundant information.

\section{Classification}

Random Forest (RF) is a supervised machine learning algorithm implemented to classify features acquired from the previous section. As an extension of Decision Tree techniques (DT) [16], RF evaluates each input considering a hierarchical set of rules randomly defined in the nodes of each tree. In this case, each window containing principal component parameters is evaluated by 250 estimators or trees, creating an illusion of a forest. As an upside-down tree, the process begins at the root node of each tree where the input is evaluated under predefined rules. Then, considering the characteristics of each sample it is conducted through branches and finally classified in the leaf nodes. Subsequently, individual decisions of all trees are compared and the mode is giving as a result of the classification problem. This characteristic makes RF methods less susceptible to overfit the model when compared with DT [16].

Using RF in classification problems, two criteria are commonly implemented to evaluate the effectiveness of a rule applied to the dataset in each node: Gini impurity and Entropy [17]. The first one is the probability of classifying incorrectly the dataset, where 1 is a highly incorrect classification and 0 represents a set of samples correctly classified. Additionally, entropy measures the randomness of the dataset in each node. For instance, a dataset with mixed samples will have high entropy since there is not a clear output for the classifier and vice versa. Evaluating the difference between the entropy before and after splitting the data is known as information gain. Information gain is required to be high to reduce the randomness of the data in fewer steps, thus making the system to converge faster [17].

Using entropy $(\mathrm{H})$ as a measure to determine how well organised are the features, the aim of the RF method is to obtain zero entropy at each node using equation 2 [17].

$$
\mathrm{H}_{\mathrm{i}}=-\sum \mathrm{P}_{\mathrm{ik}}{ }^{*} \log _{2}\left(\mathrm{P}_{\mathrm{ik}}\right), \mathrm{P}_{\mathrm{ik}} \neq 0
$$


Where entropy of the $i_{\text {th }}$ node is defined as a function of the binary $\log$ of the ratio $(\mathrm{P})$ of the $\mathrm{k}_{\text {th }}$ class.

\section{E. Evaluation Metrics}

EV charging and EV disconnected are the targets defined for the random forest classifier. Comparing input predictions with actual data, four different results can be obtained: true positive (TP), false positive (FP), true negative (TN) and false negative (FN). TP and TN represent correct predictions of EV charging and EV disconnected, respectively. FP depicts a window classified as EV charging while the actual state was disconnected. In contrast, FN stands for misclassification of EV disconnected. These parameters are combined together to form typical performance metrics used to evaluate NILM [18]. Precision (Pr), Accuracy $\left(\mathrm{A}_{\mathrm{CC}}\right)$, Recall and F1 Score $\left(\mathrm{F}_{1}\right)$ are the ones used in this case as presented in Table I.

TABLE I. EVALUATION METRICS

\begin{tabular}{|c|c|}
\hline Metric & $\mathrm{Pr}=\frac{\mathrm{TP}}{\mathrm{TP}+\mathrm{FP}}$ \\
\hline Precision & $\mathrm{A}_{\mathrm{CC}}=\frac{\mathrm{TP}+\mathrm{TN}}{\mathrm{TP}+\mathrm{FP}+\mathrm{TN}+\mathrm{FN}}$ \\
\hline Accuracy & Recall $=\frac{\mathrm{TP}}{\mathrm{TP}+\mathrm{FN}}$ \\
\hline Recall & $\mathrm{F}_{1}=\frac{2 * \mathrm{TP}}{2 * \mathrm{TP}+\mathrm{FP}+\mathrm{FN}}$ \\
\hline F1 Score & $=\frac{(6)}{}$ \\
\hline
\end{tabular}

\section{DISCUSSION}

About $12 \%$ of the input data acquired from the "Pecan Street - Dataport" dataset was used to generate balanced conditions to train and test the classifier. Therefore, $100 \%$ of the generated windows contained EV charging and about $6 \%$ containing aggregated power consumption of conventional residential loads were used during the analysed scenarios. Then, the pre-processed data was divided in training and test sets, formed by $80 \%$ and $20 \%$ of the selected data respectively.

Additionally, several parameters can be configured in the RF algorithm such as the number of estimators and the criterion hyperparameter. First, diverse tests were carried out implementing a varied number of estimators, obtaining slight improvements in the performance of the classifier as they were increased but enlarging considerably the processing times. Therefore, estimators were defined as 500, representing a balance between performance and algorithm running times. Secondly, comparing the system performance using the defined number of estimators, Gini and Entropy were evaluated as criterion hyperparameters. Since this parameter does not influence the performance of the RF method, Entropy was selected as the impurity measure of the proposed algorithm [17].
Bearing in mind the described algorithm setup, two main scenarios were assessed to test the performance of the system using pre-processed data and PCA components as inputs of the random forest classifier.

\section{A. Scenario I}

Initially, the system performance was developed using directly windows of 10 minutes width as inputs of the classifier. The number of observations during training and testing stages were 743.018 and 185.756 respectively. From the test data, 92.878 windows contained aggregated active power from conventional loads and the same proportion contained Electric Vehicle charging, thus, keeping the dataset balanced and independent from the training set. The result obtained is shown in Table II, using a confusion matrix where the total number of correct predictions outweighed the wrong classifications by more than $93 \%$ for both categories.

TABLE II. CONFUSION MATRIX

\begin{tabular}{|c|c|c|}
\hline Predicted & $\begin{array}{c}\text { EV } \\
\text { Disconnected }\end{array}$ & EV Charging \\
\hline $\begin{array}{c}\text { EV } \\
\text { Disconnected }\end{array}$ & 85.711 & 7.167 \\
\hline EV Charging & 6.610 & 86.268 \\
\hline
\end{tabular}

\section{B. Scenario II}

Considering the baseline set by the direct implementation of measurements in the classifier, the feature projection stage was applied to improve the performance of the system as discussed in section III-C. Principal components of each window were used as features, thus, ten principal components where used due to the window width. In this case, true negative and false positive values remained the same. However, better classification of EV charging was achieved and consequently, the number of false-negative classifications was reduced 138 times. Second scenario predictions are provided in Table III.

TABLE III. CONFUSION MATRIX

\begin{tabular}{|c|c|c|}
\hline Predicted & $\begin{array}{c}\text { EV } \\
\text { Disconnected }\end{array}$ & EV Charging \\
\hline $\begin{array}{c}\text { EV } \boldsymbol{V} \\
\text { Disconnected }\end{array}$ & 85.711 & 7.167 \\
\hline EV Charging & 6.472 & 86.406 \\
\hline
\end{tabular}

\section{Comparison}

Using equations (3) to (6), evaluation of precision, accuracy, recall and $\mathrm{F}_{1}$ score were carried out over the two system configurations as shown in Table IV. It can be seen how the implementation of the principal components slightly improved the classifier performance compared with the initial scenario where windows were used as direct inputs of the classifier.

TABLE IV. METRICS OBTAINED PER SCENARIO

\begin{tabular}{|c|c|c|c|c|}
\hline Scenario & $\operatorname{Pr}(\%)$ & $\mathbf{A}_{\mathbf{C C}}(\mathbf{\%})$ & Recall (\%) & $\mathbf{F}_{\mathbf{1}}(\mathbf{\%})$ \\
\hline I & 92.33 & 92.58 & 92.88 & 92.61 \\
\hline II & 92.34 & 92.66 & 93.03 & 92.69 \\
\hline
\end{tabular}




\section{CONCLUSION AND FUTURE WORK}

Migrating from fossil fuels to eco-friendly systems relying on renewables energies not only presents worldwide opportunities but also new technical challenges [19]. With electrification of the transport system, increasing the electric vehicle population modifies electrical network dynamics requiring innovative methods to ensure a smooth integration of these low carbon loads in the distribution section.

Non-intrusive load monitoring algorithms have been proposed in management energy systems, mainly focusing the targets to identify conventional electrical loads in the residential sector. In this paper, a simple classification technique was proposed to identify electric vehicles from aggregated active power measurements using the "Pecan Street - Dataport" Dataset. Samples are taken every minute over a two-year period from 6 houses and 6 EVs located in the United States of America. Data were split into 10-minute windows shifted every half width in order to analyse all power signal variations between windows.

Advantages of the proposed method rely first on the simplicity of the classification algorithm using principal components as inputs of the Random Forest machine learning algorithm. Principal components were used to reduce the dimensionality of the input data which improved the performance of the system, achieving an accuracy of $92.69 \%$. This result improves upon the reported metrics achieved by authors in [7], where ICA was implemented, to decompose aggregated power measurements reaching an overall F1 score of $89.94 \%$ for 10 -minute windows.

Furthermore, the availability of low sample-rate measurements, through existing smart metering, represents an opportunity to include identification of low carbon loads in the residential sector. This enables the integration of distributed energy resources as well as increasing flexibility and reliability of the distribution network. In addition to the cost reduction of using existing sensors, the use of low sample-rate measurements also minimises computational and storage capacity requirements compared with high-frequency sampling-rate techniques [20].

As future work, the authors plan to investigate the benefits of having data at a higher sampling-rate, in order to expand window information and reduce identification time. This will ultimately be more difficult and expensive to implement in practice but would lead to having real-time NILM electric vehicle identification which could enable advanced grid stability control systems such as staggered EV charging.

\section{ACKNOWLEDGMENT}

This work is part of the Collaborative REsearch of Decentralization, ElectrificatioN, Communications and Economics (CREDENCE) project, which is funded by a USIreland, Department for the Economy (DfE) Research, Science Foundation Ireland (SFI), National Science Foundation (NSF) and Development Partnership Program (Centre to Centre) award (grant number USI 110).

\section{REFERENCES}

[1]
A. Negi and M. Mathew, "Study on Sustainable Transportation Fuels Based on Green House Gas Emission Potential," 2018 Int. Conf. Power Energy, Environ. Intell. Control. PEEIC 2018, pp. 420-424, 2019.

H. Kaushik, M. Mathew, and J. Hossain, "Comparative Analysis of Green House Gas Emission from Different Vehicular Fuels," 2018 Int. Conf. Power Energy, Environ. Intell. Control. PEEIC 2018, pp. 702-706, 2019.

K. Mahmud and G. E. Town, "A review of computer tools for modeling electric vehicle energy requirements and their impact on power distribution networks," Appl. Energy, vol. 172, pp. 337-359, 2016.

S. Khan, A. F. Latif, and S. Sohaib, "Low-cost real-time nonintrusive appliance identification and controlling through machine learning algorithm," in International Symposium on Consumer Technologies, ISCT 2018, 2018, pp. 32-36.

A. Zoha, A. Gluhak, M. A. Imran, and S. Rajasegarar, "Nonintrusive Load Monitoring approaches for disaggregated energy sensing: A survey," Sensors, vol. 12, no. 12, pp. 16838-16866, 2012.

Pecan Street Inc., "Pecan Street Dataport," 2019. [Online]. Available: https://www.pecanstreet.org/. [Accessed: 01-Dec-2019].

A. A. Munshi and Y. A. R. I. Mohamed, "Unsupervised Nonintrusive Extraction of Electrical Vehicle Charging Load Patterns," IEEE Trans. Ind. Informatics, vol. 15, no. 1, pp. 266-279, 2019.

A. U. Rehman, T. T. Lie, B. Valles, and S. R. Tito, "Low Complexity Non-Intrusive Load Disaggregation of Air Conditioning Unit and Electric Vehicle Charging," in IEEE Innovative Smart Grid Technologies - Asia (ISGT Asia), 2019, pp. 1-6.

J. Zhu et al., "Electric Vehicle Charging Load Forecasting: A Comparative Study of Deep Learning Approaches," Energies, vol. 12, no. 14, p. 2692, 2019.

P. Held, S. Mauch, A. Saleh, and D. Benyoucef, "HELD1: Home Equipment Laboratory Dataset for Non-Intrusive Load Monitoring," Signal 2018 Third Int. Conf. Adv. Signal, Image Video Process., no. c, pp. 15-20, 2018.

K. Anderson, A. F. Acneanu, D. Benitez, D. Carlson, A. Rowe, and M. Berges, "BLUED: A fully labeled public dataset for even-based non-intrusive load monitoring research," Comput. Sci., no. October 2011, 2012.

J. Kelly and W. Knottenbelt, "UK-DALE: A dataset recording UK Domestic Appliance-Level Electricity demand and whole-house demand," Sci. Data, vol. 2, pp. 1-4, 2015.

J. Z. Kolter and M. J. Johnson, "REDD: A Public Data Set for Energy Disaggregation Research," in SustKDD workshop on Data Mining Applications in Sustainability, 2011, no. 1, pp. 1-6.

S. Raurale, J. McAllister, and J. M. Del Rincon, "Emg acquisition and hand pose classification for bionic hands from randomly-placed sensors," ICASSP, IEEE Int. Conf. Acoust. Speech Signal Process. - 
Proc., vol. 2018-April, pp. 1105-1109, 2018.

[15] S. Marsland, "Dimensionality Reduction," in Machine learning : an algorithmic perspective, 2nd ed., L. Taylor \& Francis Group, Ed. Boca Raton, FL, USA: Taylor \& Francis Group, LLC, 2015, pp. 129-130,133-140.

[16] W. Hedén, "Predicting Hourly Residential Energy Consumption using Random Forest and Support Vector Regression: An Analysis of the Impact of Household Clustering on the Performance Accuracy,” KTH Royal Institute of Technology, 2016.

[17] A. Géron, Hands-On Machine Learning with Scikit-Learn and TensorFlow, 1st ed. Sebastopol, United States of America: O’Reilly Media, 2017.
[18] M. Zhuang, M. Shahidehpour, and Z. Li, "An Overview of NonIntrusive Load Monitoring: Approaches, business Applications, and Challenges," in International Conference on Power System Technology (POWERCON), 2018, pp. 4291-4299.

[19] M. Henderson, "Renewable Energy Growth: Advantages, Challenges, and Opportunities [From the Editor]," IEEE Power Energy Mag., vol. 17, no. 6, pp. 4-8, 2019.

[20] M. Nardello, M. Rossi, and D. Brunelli, "An Innovative CostEffective Smart Controlling system," Dep. Ind. Eng. DII, Univ. Trento, Italy, p. 6, 2017. 